\title{
SPLENIC LEIOMYOMA IN DOG
}

\author{
Magdalena Magas ${ }^{1 *}$, Katarzyna Szczepańska², Maurycy Jankowski ${ }^{3}$, Dorota Bukowska ${ }^{1}$, Paweł \\ Antosik $^{1}$
}

\begin{abstract}
Leiomyoma is a benign tumour, originating from smooth muscles cells. This tumor commonly involves the uterus, vagina, stomach, intestine, urinary bladder and other organs. Only a few cases of splenic leiomyoma in dogs have been reported in the available literature. Much more frequently malignant leiomyosarcoma was found. The aim of this study was to compile rare clinical case of splenic leiomyoma in dog, which developed with no clinical signs and no abnormalities in blood findings. A 14-year-old, spayed bitch was examined with ultrasonography, where lesions on the spleen were identified. Based on the clinical findings (blood test in norm, no metastases in X-ray examination) surgical removal of spleen was recommended. Two fragments of tumors were prepare for histopathological examination. The lesion was described as smooth muscle benign tumor, therefore a diagnosis of leiomyoma was made. About a year after splenectomy no signs of metastases were present in a ultrasound and X-ray examinations. This report indicates the necessity of taking the occurrence of benign lesions in the spleen into account. Splenectomy based on the presence of tumor lesion should be associated with histopathological examination to identify the nature of change. This clinical case, despite a marked morphological lesion shown during intraoperative examination, was benign with successful prognosis.
\end{abstract}

Running title: Splenic Leiomyoma in Dog

Keywords: leiomyoma, splenic masses, splenic benign tumors, splenectomy, canine, smooth muscle

\footnotetext{
${ }^{1}$ Centre for Veterinary Sciences, Nicolaus Copernicus University in Torun, Torun, Poland

${ }^{2}$ Institute of Veterinary, Poznan University of Life Sciences, Poznan, Poland

${ }^{3}$ Department of Anatomy, Poznan University of Medical Science, Poznan, Poland

* Correspondence: magdalena.magas@gmail.com

Full list of author information is available at the end of article 


\section{Introduction}

Leiomyoma is a benign tumour, belonging to the group of mesenchymal cancers, originating from smooth muscles cells. It is rarely diagnosed in dogs and cats. Leiomyomas commonly involve the uterus, vagina, stomach, intestine, urinary bladder and other organs [1]-[3]. Cases of leiomyoma were also reported in dog oesophagus [4]. Rarely this types of tumors can be localized in the kidney [5], the third eyelid [6] or the heart [7]. In women of reproductive age, leiomyoma is one of the most common tumors of the myometrium, but the etiology of these tumors is not understood [8], [9].

Leiomyoma found in uterus, both women and animals, can be situated below endometrium (leiomyoma submucosum), below serous membrane (leiomyoma subserosum) or inside myometrium (leiomyoma intramurale). It can often take a polyp shape and extend into vaginal lumen (leiomyoma nascens) [2].

Leiomyosarcoma is malignant tumor, slow to metastasize and slow - growing cancer. It is the most common intestinal sarcoma in dogs and the 2-nd most common canine intestinal tumor. This type of tumors commonly affect older dogs (median age - 10,5 years) and involve the cecum and jejunum. Treatment for leiomyosarcoma is surgical excision, with median survival time of 12 months [10].

Uterine leiomyomas in dogs are noticed mainly in older females, especially multiparous. They are usually located in myometrium (leiomyoma intramurale). Hormonal disorders, mainly estrogen stimulation, are a factor that promotes the formation of uterus leiomyoma in bitches. This is often associated with ovarian cysts [1]. Development and growth of leiomyoma depends upon ovarian steroids but the exact mode of influence is not known. Additionally, human chorionic gonadotropin may participate in leiomyoma development in pregnant women. Some leiomyomas have a genetic etiology [8]. Clinical signs of leiomyoma are not specific and may be poorly expressed. In this regard, the surgical excision is the only used treatment [1].

Leiomyoma is constructed of myocytes and is rich in collagen masses, elastic and reticular fibers. It often appears as a fibroleiomyoma [8]. Usually, it exhibits small amounts of stromal connective tissue (leiomyoma molle), occasionally in firm formations (leiomyoma durum). While analyzing histological samples, one can observe multidirectional arrangement of muscle fibers, that are characteristic for leiomyomas which contrast with normal, healthy smooth muscle tissue that has regular structure [2]. Proliferating smooth muscle cells are surrounded by pseudo-capsule and the leiomyoma mass demonstrates much more deposition into the extracellular matrix (more collagen than normal smooth muscle tissue) [8]. The origins of leiomyoma are most probably the undifferentiated perivascular cells, or the muscular layer of organ in which it occurs. The retrograde changes are often observed within leiomyoma, such as necrosis, hyalinization and mucoid degeneration [2].

Splenic leiomyomas in dogs are rarely diagnosed. Spanglerm, Culbertson and Kass have published studies, which aim was to characterize rare tumors of the spleen. They excluded the most common neoplasms of spleen, such as hemangiosarcoma, and selected eighty seven neoplasms of the spleen considered to be of nonangiomatous and nonlymphomatous. Only three cases of leiomyoma were reported. Additionally, the presence of malignant leiomyosarcoma was more common (14/87) [11]. Further research showed one case of dog with leiomyosarcoma, among 25 splenic masses, leiomyoma was not diagnosed [12]. Eberle et al. retrospectively described clinical aspects and histopathologic characteristics of dogs with splenic masses. They included 249 dogs, where there were no cases of leiomyoma, and only one case of leiomyosarcoma [13].

The splenectomy in dogs is often and commonly performed in veterinary institutions. The high availability of ultrasonography allows early diagnoses of spleen masses (either generalized splenomegaly or focal splenic tumors), what can be a therapeutic indication for splenectomy. Splenic lesions can be benign (hemangioma, leiomyoma, hematoma, abscess, nodular hyperplasia, cysts) or malignant (hemangiosarcoma - most common, leiomyosarcoma, lymphosarcoma). It is not possible to differentiate the type of lesion with common methods (either USG), which could help determine the prognosis and establish treatment [12], [14].

\section{Materials and Methods}

A 14-year-old, spayed, mixed breed bitch, weighing $24 \mathrm{~kg}$, was presented to veterinary hospital to routine examination. The dog did not show any disease symptoms, despite this, a clinical trial was performed, including ultrasonography and laboratory tests of blood. Full blood count performed by using Mythic 18 Orphee, biochemistry serum tests - Pointe 180 . On physical examination, the animal appeared healthy with no abnormalities. The rectal temperature, respiratory rate and pulse rate were all within normal ranges. Palpation revealed no generalized rigidity, splenomegaly or pain in abdominal area. Exclusively, ultrasound revealed an irregular shaped mass on the spleen.

Ultrasound examination (Logiq Book; $10 \mathrm{MHz}$ ) of the abdomen identified an irregularly shaped, heteroechogenic lesion on body of the spleen. This lesion, size: 4,7 x 4,4 cm (Fig. 1), showed signs of increased vascularization in Doppler examination. Fat around the lesion was reactive (hyperechoic). There was also a second lesion in the cranial end of spleen, oval-shaped, size: $1,5 \mathrm{~cm}$, but low vascularization. The spleen exhibited general magnification, with rounded edges. The rest of organs in the ultrasound examination did not show any abnormalities. 


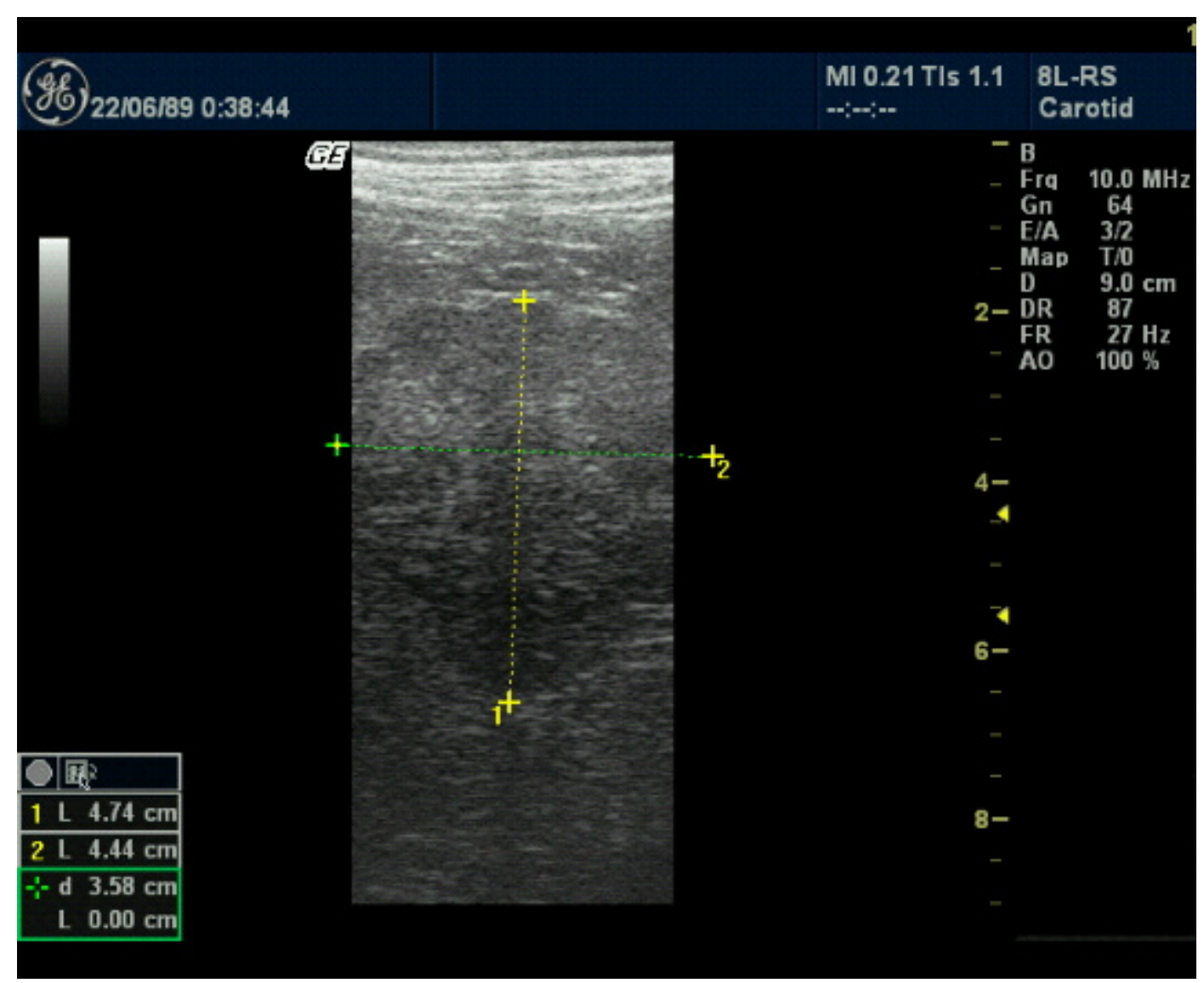

Figure 1 Ultrasonographic image of splenic tumor

The hematological and serum biochemistry findings were within normal ranges. The X-ray examination of abdomen and chest was performed to exclude the presence of metastases.

Based on the clinical findings (including blood tests, ultrasonographic and radiological results) a tentative diagnosis of splenic tumor was made. Surgical removal of spleen was recommended.

\section{Surgical procedure}

The dog was pre-medicated with $40 \mu \mathrm{g} / \mathrm{kg}$ intramuscular medetomidine (Domitor $\AA$ - Orion Corporation, Finland). General anesthesia was maintained with $10 \mathrm{mg} / \mathrm{kg}$ ketamine (Bioketan $\AA$ - Vetoquinol, France). The dog was prepared to surgery (operative field shaved, defatted with alcohol and painted with iodine). A laparotomy was performed via a midline incision from the xiphoid process of sternum to umbilicus. After abdominal section the splenic lesion (Fig. 2) has become observable. Proper blood vessels were ligated and splenectomy was performed. The abdominal cavity was examined for evidence of hemorrhaging and metastases. Any signs of metastases in other organs were not revealed. Thereafter the peritoneal cavity and muscles were closed with running suture (Vicryl 0), subcutaneous tissue with running suture (Vicryl 2 -0). Integumentum commune was closed with horizontal mattress suture (Vicryl $2-0$ ). The surgical wound was cleaned with hydrogen peroxide solution and covered with Alu Spray®. Both of splenic masses were excised in a routine manner and submitted for histological evaluation. Post - operative treatment included administration of intravenous fluid therapy (Solution Ringeri), a prophylactic antibiotic (amoxycilin with clavulanic acid), analgesic and anti-inflammatory drugs (NSAID - meloxicam, metamizole sodium). The bitch recovered well from the surgery . An Elizabethan collar was placed on the dog and it was sent home after the surgical procedure. Two fragments of tumors were fixed in $10 \%$ buffered formalin, embedded in paraffin wax, sectioned at $4 \mu \mathrm{m}$ and then stained with hematoxylin and eosin (H\&E) for histopathological examination. A few days after surgery the bitch was bright and active and had no problems with defecation. The suture was removed 10 days after surgery, the wound was healed by first intention.

Ethical approval: The research related to animals use has been complied with all the relevant national regulations and institutional policies for the care and use of animals. Animal Ethics Committee approval no. 83/2012/DNT.

\section{Results}

The lesion was described as smooth muscle, benign tumor (Fig. 3). Therefore, a diagnosis of leiomyoma was made. The mitotic activity and cellular 


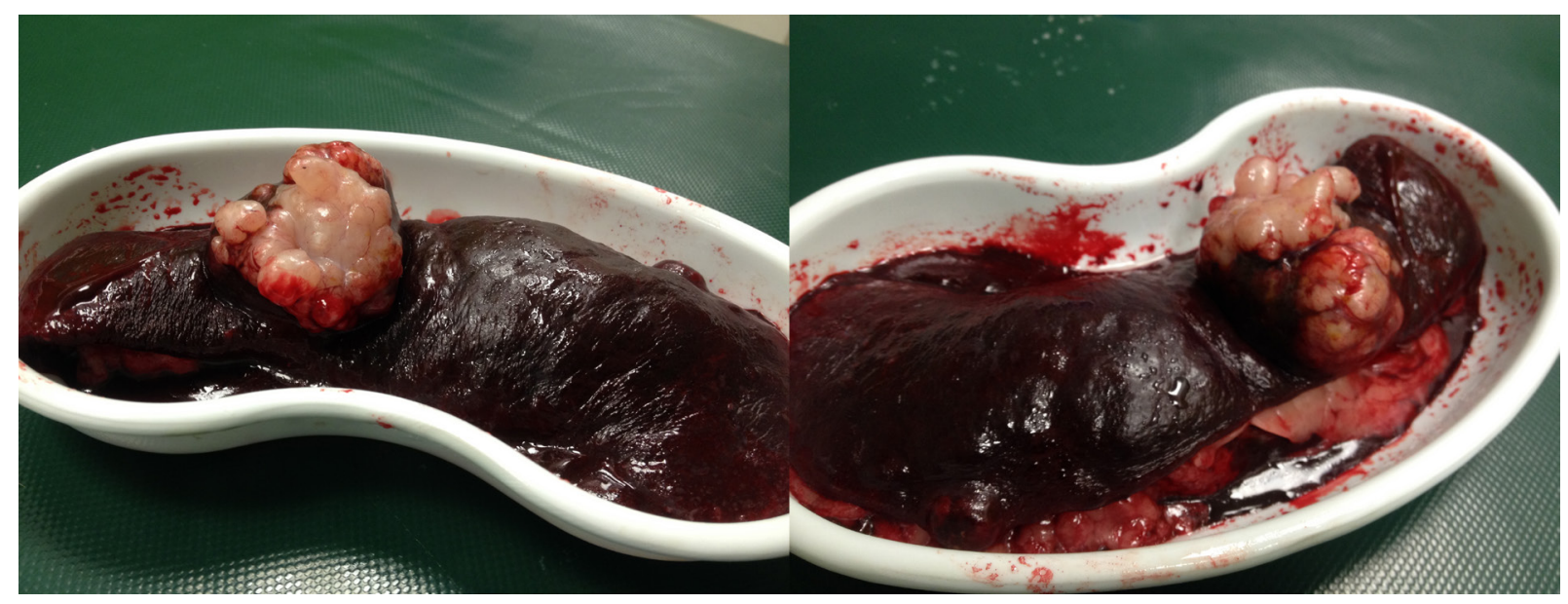

Figure 2 The spleen after splenectomy with tumors

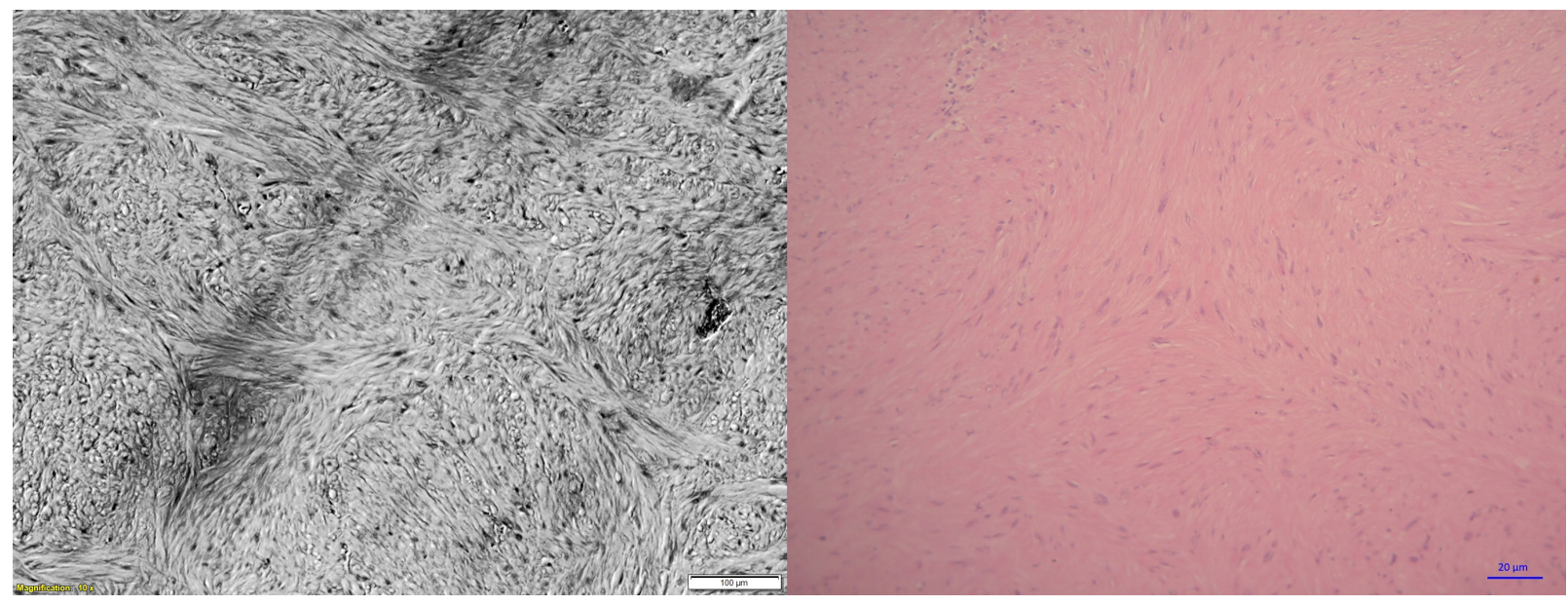

Figure 3 Photomicrograph of leiomyoma mass (multidirectional arrangement of muscle fibers)

atypia were not found, necrosis was revealed. The structure of spleen was analysed at the circumference of the change. No histological features or malignancies were found. The middle layer (tunica media) of splenic blood vessels was probably the source of the leiomyoma in this case.

Twelve months after the surgery, bloods tests were made, where there was no abnormalities. Additionally, the ultrasonography of abdomen was performed, especially intestines and liver, but no signs of metastases were present.

\section{Discussion}

Splenic leiomyomas are infrequently reported in dogs. This tumor was diagnosed based on histopathology. Spanglerm et al. described rare mesenchymal splenic neoplasms in 1994. They excluded the most popular neoplasms - angiomatous and lymphomatous (e.g. hemangiosarcoma). The aim of this research was to classify splenic neoplasms of 87 dogs. Leiomyosarcoma was diagnosed in 14 dogs, but leiomyoma only in 3 cases. Mean survival time (after splenectomy) in dogs with leiomyosarcoma evaluated in 6 months [11]. Another research in- volved 25 dogs with splenic tumors, where 16 cases were diagnosed as malignant neoplasms, among which only one case revealed as leiomyosarcoma (10 years old, neutered golden retriever, surgery shown metastases in liver). Leiomyoma was not found [12]. More recent reports published in 2012 involved the lesions of spleen, indicate a similar percentage of malignant to benign lesions (53:47\%). In group of 249 dogs, 132 malignant neoplasms were diagnosed, among which the most common was haemangiosarcoma (97 cases - 73,5\%), in turn the rarest leiomyosarcoma ( 1 case - 0,4\%). The leiomyoma was also not found [13].

\section{Conclusions}

Bearing in mind that the splenic leiomyoma is rare disease in dogs and clinical signs may not be present, we have made a decision to compile this case study. This report indicates the necessity of taking the occurrence of benign lesions in the spleen into account, as early detection and removal of this kind of tumors the leaves patient able to live for a relatively long time, without further implications to physical health and well-being. It has also shown 
a high value of routine ultrasound examinations to exclude or detect such lesions.

Any splenectomy based on the presence of tumor lesion should be associated with histopathological examination. Identification of the nature of the change with the basic methods, commonly available in veterinary practices (X-ray, USG) is difficult and vague. About half of splenic lesions are malignant. The most common malignant cancer in dogs is haemangiosarcoma, for which the survival prognoses are usually poor [12]. Aspiration biopsy of spleen is not a good diagnostic method as it may not be sufficiently representative [13]. In addition, aspiration of the material may result in the accidental scattering of tumor cells in the body [12]. This clinical case, despite a marked morphological lesion shown during intraoperative examination, was benign with successful prognosis.

\section{Acknowledgements}

The authors thank dr Bartosz Kempisty for help in manuscript preparation

\section{Author details}

Magdalena Magas, Centre for Veterinary Sciences, Nicolaus Copernicus University in Toruń, Toruń, Poland, tel.: +48 668611416, e-mail: magdalena.magas@gmail.com

\section{Conflicts of Interest}

The authors declare they have no conflict of interest

\section{References}

1. Dąbrowski, R., Szachowicz, A., Brodzki, P., and Śmiech, A., "Leiomyoma u suki - opis przypadku," Weter. w Prakt., vol. 8, pp. 56-58, 2011

2. Madej, J. A. and Rotkiewicz, T., Patologia ogólna zwierząt. Olsztyn: Wydawnictwo Uniwersytetu Warmińsko - Mazurskiego w Olsztynie, 2006.

3. Sapierzyński, R., Onkologia praktyczna psów i kotów. Wrocław: Elsevier Urban \& Partner, 2010.

4. Madej, J. A., Rotkiewicz, T., and Nozdryn-Płotnicki, Z., Patologia szczegółowa zwierząt. Olsztyn: Wydawnictwo Uniwersytetu Warmińsko - Mazurskiego w Olsztynie, 2007.

5. Laluha, P., Grest, P., Eichenberger, S., Reusch, C. E., and Gerber, B., "Leiomyom einer Niere beim Hund - eine seltene Diagnose," Schweiz Arch Tierheilkd, vol. 148, no. 6, pp. 303-307, 2006

6. Mathes, R. L., Noble, S. J., and Ellis, A. E., "Leiomyoma of the third eyelid in a dog," Vet. Ophthalmol., vol. 19, no. 4, pp. 347-354, 2016.

7. Gallay, J., Bélanger, M. C., Hélie, P., Côté, E., Johnson, T. O., and Peters, M. E. "Cardiac leiomyoma associated with advanced atrioventricular block in a young dog," J. Vet. Cardiol., vol. 13, no. 1, pp. 71-77, 2011.

8. Sarais, V. et al., "Human Chorionic Gonadotrophin as a Possible Mediator of Leiomyoma Growth during Pregnancy: Molecular Mechanisms," Int. J. Mol. Sci., vol. 18, no. 9, 2017.

9. Holdsworth-Carson, S. J., Zaitseva, M., Vollenhoven, B. J., and Rogers, P. A W., "Clonality of smooth muscle and fibroblast cell populations isolated from human fibroid and myometrial tissues," Mol. Hum. Reprod., vol. 20 no. 3, pp. 250-259, 2014

10. Cohen, M., Post, G. S., and Wright, J. C., "Gastrointestinal leiomyosarcoma in 14 dogs.," J. Vet. Intern. Med., vol. 17, no. JANUARY 2003, pp. 107-110, 2003.

11. Spangler, W. L., Culbertson, M. R., and Kass, P. H., "Primary mesenchymal (nonangiomatous /nonlymphomatous) neoplasms occurring in the canine spleen: anatomic classification, immunohistochemistry, and mitotic activity correlated with patient survival.," Vet. Pathol., vol. 31, no. 1994, pp. 37-47, 1994

12. Irausquin, R. A. et al., "Comparative evaluation of the liver in dogs with a splenic mass by using ultrasonography and contrast-enhanced computed tomography.", Can. Vet. J., vol. 49, no. 1, pp. 46-52, 2008.

13. Eberle N., Babo, V. Von, Nolte, I., Baumgärtner, W., and Betz, D., "Splenic masses in dogs," Tpk.Schattauer.De, vol. 40, no. 10, pp. 250-260, 2012
14. Glińska-Suchocka, K., Jankowski, M., Borusewicz, P., Spużak, J., and Kubiak, K., "Choroby śledziony - cz. II. Niesymetryczne powiększenie narzadu.," Weter. w Prakt., vol. 11, pp. 41-45, 2014. 